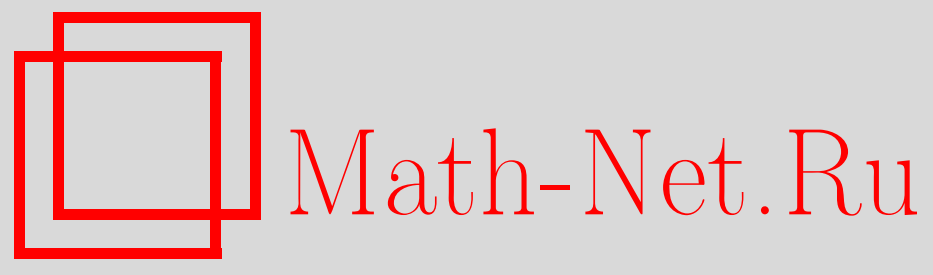

Л. Д. Пустыльников, Дискретные волновые уравнения со случайными параметрами и дискретная струна со случайными массами, Теория вероятн. и ее примен., 2002, том 47, выпуск 2, 255-269

DOI: https://doi.org/10.4213/tvp3646

Использование Общероссийского математического портала MathNet.Ru подразумевает, что вы прочитали и согласны с пользовательским соглашением

http://www . mathnet.ru/rus/agreement

Параметры загрузки:

IP : 54.198 .55 .26

26 апреля 2023 г., 06:29:17

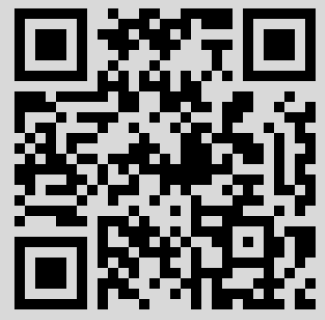




\title{
ДИСКРЕТНЫЕ ВОЛНОВЫЕ УРАВНЕНИЯ СО СЛУЧАЙНЫМИ ПАРАМЕТРАМИ И ДИСКРЕТНАЯ СТРУНА СО СЛУЧАЙНЫМИ МАССАМИ
}

\begin{abstract}
Рассматриваемая проблема является современным развитием одной из самых старых задач математики и механики об исследовании колебаний струны, масса которой сосредоточена в конечном числе равноудаленных друг от друга точек, на случай, когда массы точек представляют собой реализации последовательности случайных величин. На основе изучения соответствующего дифференциального уравнения со случайными параметрами получены явные асимптотические выражения для частот и амплитуд случайных колебаний и их вероятностных характеристик для струны конечного размера и в ситуации, когда число точек стремится к бесконечности. Доказаны центральные предельные теоремы для функций, характеризующих частоты колебаний.

Ключевые слова и фразы: дискретная струна, случайные массы, дискретное волновое уравнение со случайными параметрами, частоты колебаний, амплитуды колебаний.
\end{abstract}

Введение. Изучаемая проблема является современным развитием одной из самых старых задач математики и механики: исследования колебаний струны, масса которой сосредоточена в конечном числе равноудаленных друг от друга точек. Эта проблема была предметом пристального внимания со стороны Эйлера, Даламбера, Д. Бернулли, Лагранжа, Штурма и многих других ученых и оказала огромное влияние на развитие других разделов математики и механики (см. [1], [2]). Классиками был подробно и окончательно изучен частный случай этой задачи, когда массы всех точек - одинаковые, и для этого случая были найдены явные формулы для частот и амплитуд колебаний (теорема A, п. 1). Общий же случай произвольных масс (неоднородная дискретная струна) указывался в качестве задачи (см. [1]) и был детально изучен в работе [3], где были получены явные выражения для решений соответствующих дифференциальных уравнений и, как следствие, - для частот и амплитуд колебаний неоднородной дискретной струны (теоремы B, C, п. 1).

${ }^{*}$ Институт прикладной математики им. М. В. Келдыша РАН. 
В настоящей работе, в связи с бурным развитием теории стохастических дифференциальных уравнений и их многочисленными применениями, постановка указанной проблемы естественным образом расширяется на еще более общий случай, когда массы точек, составляющих струну, представляют собой реализации последовательности случайных величин. В этом случае частоты и амплитуды колебаний также являются случайными величинами, и основная задача исследования в этой постановке - нахождение вероятностных характеристик этих величин (математических ожиданий, ковариаций и др.) как для струны фиксированного размера (число точек - фиксировано), так и в ситуации, когда число точек стремится к бесконечности. С математической точки зрения задача сводится к изучению свойств решений дискретного волнового уравнения

$$
m_{n} \frac{d^{2} q_{n}}{d t^{2}}-T\left(q_{n+1}-2 q_{n}+q_{n-1}\right)=0 \quad(n=1, \ldots, N-1)
$$

с граничными условиями

$$
q_{0}(t)=q_{N}(t) \equiv 0
$$

в котором константа $T>0$ характеризует натяжение струны, а константы $m_{n}(n=1,2, \ldots)$, характеризующие массы точек, суть реализации последовательности положительных случайных величин.

Работа состоит из пяти пунктов. В п. 1 сформулированы основные используемые в дальнейшем результаты, касающиеся уравнения (1) с фиксированными параметрами. В пп. 2-4 предполагается, что массы $m_{n}$ имеют вид $m_{n}=m+\varepsilon \xi_{n}$, где $m>0, \varepsilon$ - положительный параметр, меняющийся на отрезке $0 \leqslant \varepsilon \leqslant 1$, а $\xi_{n}(n \geqslant 1)$ - последовательность взаимно независимых случайных величин. При этих предположениях в п. 2 получены явные асимптотические (относительно параметра $\varepsilon$ ) выражения для частот и амплитуд случайных колебаний и их основных вероятностных характеристик, в п. 3 доказана основная теорема и ее следствия, дающие асимптотики при $N \rightarrow \infty$ случайных величин, характеризующих частоты и амплитуды колебаний, а в п. 4 доказана центральная предельная теорема для бесконечной последовательности случайных величин, характеризующих частоты. Доказательство Основной теоремы (п. 3) базируется на одном обобщении усиленного закона больших чисел для последовательности независимых случайных величин (теоремы 6-8 п. 3), и благодаря этому обобщению асимптотики, найденные в Основной теореме и ее следствиях 1-3, уже случайными не являются, а определяются только математическими ожиданиями случайных величин $\xi_{n}$.

Последний п. 5 посвящен изучению случайной величины $\Omega_{N}$, представляющей собой сумму квадратов всех частот, входящих в решения уравнения (1) с условием (2). В предположении, что положительные 
случайные величины $\eta_{n}=1 / m_{n}$ - взаимно независимы, здесь доказаны теорема 7 и ее следствия 4,5 , дающие асимптотику $\Omega_{N}$ при $N \rightarrow \infty$, зависяшую только от математических ожиданий величин $\eta_{n}$, а также центральная предельная теорема для последовательности $\Omega_{N}$ при $N \rightarrow \infty$ (теорема 8).

Первоначально результаты этой работы были опубликованы в [5].

Автор выражает благодарность научному центру Bielefeld-BonnStochastik (BiBoS) за поддержку.

\section{1. Явные выражения для решений уравнения с фиксиро-} ванными параметрами. В этом пункте сформулированы результаты (теоремы A, B, С) о решениях уравнения (1) с фиксированными параметрами, удовлетворяющих условию (2), которые используются в дальнейшем.

Теорема А. Предположим, что в уравнении (1) все константы $m_{n}(n=1, \ldots, N-1)$ - одинаковые и равнь числу $m>0$. Тогда общее решение уравнения (1) с условием (2) имеет вид

$$
q_{n}(t)=\sum_{k=1}^{N-1} c_{k} \sin \frac{\pi k n}{N} \sin \left(2 \sqrt{\frac{T}{m}} \sin \frac{\pi k}{2 N} t+\alpha_{k}\right) \quad(n=1, \ldots, N-1)
$$

где $c_{k}, \alpha_{k}(k=1, \ldots, N-1)$ - произвольнье константьи, зависяшие только от начальньх данньи.

Утверждение теоремы А непосредственно вытекает из следующей теоремы В, относящейся к более общему случаю, когда параметры $m_{n}$ произвольные, но близкие между собой числа.

Теорема В. Предположим, что $m_{n}-m=\varepsilon a_{n}^{0}(n=0, \ldots, N-1)$, где $\varepsilon-$ малый положительный параметр, $m>0, a_{0}^{0}=0$. Тогда общее решение уравнения (1) с условием (2) имеет вид $q_{n}(t)=$ $\sum_{k=1}^{N-1} c_{k} g_{k}(n) \sin \left(\omega_{k} t+\alpha_{k}\right)$, где $n=1, \ldots, N-1, c_{k}$ и $\alpha_{k}-$ произвольнье константы, не зависящие от $n u t$,

$$
\begin{aligned}
\omega_{k}= & 2 \sqrt{\frac{T}{m}} \sin \left\{\frac{\pi k}{2 N}-\frac{\varepsilon}{N} \operatorname{tg} \frac{\pi k}{2 N} \sum_{s=1}^{N-1}\left(\frac{a_{s}^{0}}{m_{s}} \sin ^{2} \frac{s \pi k}{N}\right)\right\}+O\left(\varepsilon^{2}\right), \\
g_{k}(n)= & \sin \left\{\frac{\pi k n}{N}+\frac{2 \varepsilon n}{N} \operatorname{tg} \frac{\pi k}{2 N} \sum_{s=1}^{N-1}\left(\frac{a_{s}^{0}}{m_{s}} \sin ^{2} \frac{s \pi k}{N}\right)\right\} \\
& -\varepsilon \operatorname{tg} \frac{\pi k}{2 N} \sum_{s=0}^{n-1}\left\{\frac{a_{s}^{0}}{m_{s}}\left(\cos \frac{\pi k n}{N}-\cos \frac{2 s \pi k}{N}\right)\right\}+O\left(\varepsilon^{2}\right),
\end{aligned}
$$

а символ $O\left(\varepsilon^{2}\right)$ означает, что $\left|O\left(\varepsilon^{2}\right)\right|<c \varepsilon^{2}$, где с-константа, не зависящая от $\varepsilon$ u .

Доказательство теоремы В дано в [3] (п. 5, теорема 7). 
Для того чтобы сформулировать теорему $\mathrm{C}$, относяшуюся к самому общему случаю, когда параметры $m_{n}$ в уравнении (1) - произвольные положительные числа, нам потребуется ввести следуюшие определения.

О п р е д е л е н и е 1 . Пусть $k$ - натуральное число. Символом $\left[k_{\sigma}, k_{\sigma-1}, \ldots, k_{0}\right]$ будет обозначаться двоичная запись числа $k$, т.е. при $s=0,1, \ldots, \sigma-1 k_{s}$ - символ, принимающий значения 0 или $1, k_{\sigma}=1$, а число $k$ представляется в виде $k=\sum_{s=0}^{\sigma} k_{s} 2^{s}$.

О п р е д е л е н и е 2. Пусть $\left[k_{\sigma}, k_{\sigma-1}, \ldots, k_{0}\right]$ - двоичная запись числа $k$. Обозначим через $\mu_{k}$ количество единиц в последовательности $k_{0}, \ldots, k_{\sigma}$, а через $d_{1}^{(k)}, \ldots, d_{\tau_{k}}^{(k)}$ обозначим все такие индексы в последовательности $1, \ldots, \sigma$, что при $s=1, \ldots, \tau_{k}$ справедливо равенство $k_{d_{s}^{(k)}}=k_{d_{s}^{(k)}-1}=0$, причем $\tau_{k}-$ их общее количество.

О п р е д е л е н и е 3 . Обозначим через $\Omega$ множество, состоящее из таких четных чисел $k>0$, у которых в их двоичной записи $\left[k_{\sigma}, k_{\sigma-1}, \ldots, k_{0}\right]$ нет двух рядом стоящих единиц, а $\tau_{k} \neq 0$.

Теорема С. При $n=1, \ldots, N$ введем многочленьи

$$
\phi_{n}(\lambda)=\sum_{\substack{k=2^{n} \\ k \in \Omega}}^{2^{n+1}-2}(-1)^{\mu_{k}-1} \prod_{s=1}^{\tau_{k}}\left(\frac{\lambda m_{d_{s}^{(k)}}}{T}+2\right)+\cos \frac{(n-1) \pi}{2} \sin ^{2} \frac{\pi n}{2},
$$

где числа $\mu_{k}, \tau_{k}, d_{1}^{(k)}, \ldots, d_{\tau_{k}}^{(k)}$ и множество $\Omega$ введень в определениях 1-3. Тогда общее решение уравнения (1) с условиями (2) имеет вид

$$
q_{n}(t)=\sum_{\phi_{N}(\widetilde{\lambda})=0} c_{\tilde{\lambda}} \phi_{n}(\tilde{\lambda}) \sin \left(\sqrt{-\tilde{\lambda}} t+\alpha_{\tilde{\lambda}}\right)
$$

где $n=1, \ldots, N-1$, суммирование распространено на все корни $\widetilde{\lambda}$ многочлена $\phi_{N}(\lambda)$, а $c_{\tilde{\lambda}}$ и $\alpha_{\tilde{\lambda}}-$ произвольнье константьи, не зависячие om $n u t$.

Доказательство теоремы С дано в [3] (п. 5, теорема 6).

2. Вероятностные характеристики случайных колебаний струны фиксированного размера. В этом пункте, а также в пп. 3 и 4 мы предполагаем, что массы $m_{n}(n \geqslant 1)$ - случайные величины такие, что $m_{n}=m+\varepsilon \xi_{n}$, где $m>0,0 \leqslant \varepsilon \leqslant 1$, а $\xi_{n}(n \geqslant 1)$ - последовательность взаимно независимых случайных величин, удовлетворяющих неравенству $m^{\prime}<m+\xi_{n}<m^{\prime \prime}, n \geqslant 1$, в котором $m^{\prime}$ и $m^{\prime \prime}$ - положительные константы, не зависящие от $\xi_{n}$ и $n$. В этом случае при любом натуральном $N \geqslant 2$ и фиксированном $\varepsilon$ решение уравнения (1) с условием (2) представляется в виде

$$
q_{n}(t) \stackrel{\text { def }}{=} q_{n, N}^{(\varepsilon)}(t)=\sum_{k=1}^{N-1} c_{k} G_{k, N}^{(\varepsilon)}(n) \sin \left(\Omega_{k, N}^{(\varepsilon)} t+\alpha_{k}\right),
$$


где $n=1, \ldots, N-1$, частоты $\Omega_{k, N}^{(\varepsilon)}$ и амплитуды $G_{k, N}^{(\varepsilon)}(n)$ колебаний случайные величины, удовлетворяющие в силу теоремы В (п. 1) равенствам

$$
\lim _{\varepsilon \rightarrow 0} \Omega_{k, N}^{(\varepsilon)}=2 \sqrt{\frac{T}{m}} \sin \frac{\pi k}{2 N}, \quad \lim _{\varepsilon \rightarrow 0} G_{k, N}^{(\varepsilon)}(n)=\sin \frac{\pi k n}{N}, \quad G_{k, N}^{(\varepsilon)}(1)=G_{k, N}^{(0)}(1),
$$

а $c_{k}$ и $\alpha_{k}(k=1, \ldots, N-1)$ - произвольные константы, не зависящие от $n$ и $t$. Введем случайные величины

$$
\omega_{k, N}^{(\varepsilon)}=\Omega_{k, N}^{(\varepsilon)}-2 \sqrt{\frac{T}{m}} \sin \frac{\pi k}{2 N}, \quad g_{k, N}^{(\varepsilon)}(n)=G_{k, N}^{(\varepsilon)}(n)-\sin \frac{\pi k n}{N} .
$$

Целью этого пункта является нахождение явных асимптотических выражений для случайных величин $\omega_{k, N}^{(\varepsilon)}, g_{k, N}^{(\varepsilon)}(n)$ и их вероятностных характеристик (математических ожиданий, ковариаций) при $\varepsilon \rightarrow 0$ и фиксированном $N$.

Если $\xi$ и $\eta$ - случайные величины, то символом $\mathbf{E} \xi$ обозначается математическое ожидание $\xi$, символом $\mathbf{D} \xi$ - дисперсия $\xi$, а символом $\operatorname{cov}(\xi, \eta)$ - ковариация $\xi$ и $\eta$, т.е. $\operatorname{cov}(\xi, \eta)=\mathbf{E}((\xi-\mathbf{E} \xi)(\eta-\mathbf{E} \eta))$.

Теорема 1. При $k=1, \ldots, N-1, n=1, \ldots, N-1$ случайные величиньл $\omega_{k, N}^{(\varepsilon)}$ и $g_{k, N}^{(\varepsilon)}(n)$ удовлетворяют следующим равенствам:

$$
\begin{aligned}
\lim _{\varepsilon \rightarrow 0} \frac{\omega_{k, N}^{(\varepsilon)}}{\varepsilon}= & -\frac{2}{N m} \sqrt{\frac{T}{m}} \sin \frac{\pi k}{2 N} \sum_{s=1}^{N-1} \xi_{s} \sin ^{2} \frac{s \pi k}{N}, \\
\lim _{\varepsilon \rightarrow 0} \frac{g_{k, N}^{(\varepsilon)}(n)}{\varepsilon}= & \frac{2 n}{N m} \cos \frac{\pi k n}{N} \operatorname{tg} \frac{\pi k}{2 N} \sum_{s=1}^{N-1} \xi_{s} \sin ^{2} \frac{s \pi k}{N} \\
& -\frac{1}{m} \operatorname{tg} \frac{\pi k}{2 N} \sum_{s=0}^{n-1} \xi_{s}\left(\cos \frac{\pi k n}{N}-\cos \frac{2 s \pi k}{N}\right),
\end{aligned}
$$

əдe $\xi_{0} \equiv 0$.

Теорема 2. При $k=1, \ldots, N-1, n=1, \ldots, N-1$ математические ожидания $\mathbf{E} \omega_{k, N}^{(\varepsilon)} u \mathbf{E} g_{k, N}^{(\varepsilon)}(n)$ удовлетворяют следуюшим равенствам:

$$
\begin{aligned}
\lim _{\varepsilon \rightarrow 0} \frac{\mathbf{E} \omega_{k, N}^{(\varepsilon)}}{\varepsilon}= & -\frac{2}{N m} \sqrt{\frac{T}{m}} \sin \frac{\pi k}{2 N} \sum_{s=1}^{N-1} \mathbf{E} \xi_{s} \sin ^{2} \frac{s \pi k}{N} \\
\lim _{\varepsilon \rightarrow 0} \frac{\mathbf{E} g_{k, N}^{(\varepsilon)}(n)}{\varepsilon}= & \frac{2 n}{N m} \cos \frac{\pi k n}{N} \operatorname{tg} \frac{\pi k}{2 N} \sum_{s=1}^{N-1} \mathbf{E} \xi_{s} \sin ^{2} \frac{s \pi k}{N} \\
& -\frac{1}{m} \operatorname{tg} \frac{\pi k}{2 N} \sum_{s=0}^{n-1} \mathbf{E} \xi_{s}\left(\cos \frac{\pi k n}{N}-\cos \frac{2 s \pi k}{N}\right),
\end{aligned}
$$

где $\mathbf{E} \xi_{0} \equiv 0$. 
Теорема 3. При $k=1, \ldots, N-1, r=1, \ldots, N-1$ ковариачии $\operatorname{cov}\left(\omega_{k, N}^{(\varepsilon)}, \omega_{r, N}^{(\varepsilon)}\right)$ удовлетворяют следуюшим равенствам:

$$
\lim _{\varepsilon \rightarrow 0} \frac{\operatorname{cov}\left(\omega_{k, N}^{(\varepsilon)}, \omega_{r, N}^{(\varepsilon)}\right)}{\varepsilon^{2}}=\frac{4 T}{N^{2} m^{3}} \sin \frac{\pi k}{2 N} \sin \frac{\pi r}{2 N} \sum_{s=1}^{N-1} \mathbf{D} \xi_{s} \sin ^{2} \frac{s \pi k}{N} \sin ^{2} \frac{s \pi r}{N}
$$

Аналогичные равенства справедливь для величин $\operatorname{cov}\left(g_{k, N}^{(\varepsilon)}(n), g_{r, N}^{(\varepsilon)}(l)\right)$ u $\operatorname{cov}\left(\omega_{k, N}^{(\varepsilon)}, g_{r, N}^{(\varepsilon)}(l)\right)$.

Теоремы 1-3 непосредственно выводятся из теоремы В (п. 1) и основ теории вероятностей [4].

3. Обобщение усиленного закона больших чисел для последовательности независимых случайных величин и асимптотическое поведение частот и амплитуд случайных колебаний при увеличении размера струны. Согласно теореме 1 , при $k=1, \ldots, N-1, n=1, \ldots, N-1$ определены случайные величины

$$
\omega_{k, N}=\lim _{\varepsilon \rightarrow 0} \frac{\omega_{k, N}^{(\varepsilon)}}{\varepsilon}, \quad g_{k, N}(n)=\lim _{\varepsilon \rightarrow 0} \frac{g_{k, N}^{(\varepsilon)}(n)}{\varepsilon}
$$

характеризующие скорость изменения величин $\Omega_{k, N}^{(\varepsilon)}$ и $G_{k, N}^{(\varepsilon)}(n)$ в $(5)$ по $\varepsilon$ при $\varepsilon=0$. Главными результатами этого пункта являются Основная теорема и ее следствия 1-3, дающие асимптотические равенства (а следствие 2 - даже полное равенство), которым удовлетворяют случайные величины $\omega_{k, N}$ и $g_{k, N}(n)$ при $N \rightarrow \infty, n \rightarrow \infty$ и фиксированном $k$. Эти равенства уже случайными не являются и определяются только математическими ожиданиями случайных величин $\xi_{n}$. В основе доказательства Основной теоремы лежат теорема 1 и обобщения усиленного закона больших чисел для последовательности независимых случайных величин, доказанные в теоремах 4,5 и 6.

Теорема 4. Пусть $\xi_{n}(n=1,2, \ldots)$ - бесконечная последовательность взаимно независимых случайньх величин, имеющих конечные математические ожидания $M_{n}=\mathbf{E} \xi_{n}$ и дисперсии $D_{n}=\mathbf{D} \xi_{n}$, удовлетворяющие неравенству $\sum_{n=1}^{\infty} D_{n} / n^{2}<\infty$, a $f(x)$ - функция на отрезке $0 \leqslant x \leqslant 1$, удовлетворяющая условию Липшииа. Тогда с вероятностью 1 справедливо равенство

$$
\lim _{n \rightarrow \infty}\left(\frac{1}{n} \sum_{k=1}^{n} f\left(\frac{k}{n}\right) \xi_{k}-\frac{1}{n} \sum_{k=1}^{n} f\left(\frac{k}{n}\right) M_{k}\right)=0 .
$$

3 а м е ч а н и е 1 . В частном случае, когда $f(x) \equiv 1$, теорема 4 есть первая теорема Колмогорова об усиленном законе больших чисел [4].

Доказательство теоремы 4. Вводя величины $\eta_{n}=$ $\xi_{n}-M_{n}$ и числа $a_{k, n}=f(k / n)$, сведем утверждение теоремы к дока- 
зательству равенства

$$
\lim _{n \rightarrow \infty} \frac{1}{n} \sum_{k=1}^{n} a_{k, n} \eta_{k}=0 .
$$

Применяя к сумме в (9) преобразование Абеля, имеем:

$$
\frac{1}{n} \sum_{k=1}^{n} a_{k, n} \eta_{k}=\sum_{k=1}^{n-1}\left(a_{k, n}-a_{k+1, n}\right) \frac{\eta_{1}+\cdots+\eta_{k}}{n}+\frac{\eta_{1}+\cdots+\eta_{n}}{n} a_{n, n} .
$$

Так как функция $f(x)$ на отрезке $0 \leqslant x \leqslant 1$ удовлетворяет условию Липшица, то справедливо неравенство

$$
\left|a_{k, n}-a_{k+1, n}\right|=\left|f\left(\frac{k}{n}\right)-f\left(\frac{k+1}{n}\right)\right|<\frac{C_{1}}{n},
$$

где $C_{1}$ - константа, зависящая только от функции $f(x)$. Поэтому в силу (10)

$$
\left|\frac{1}{n} \sum_{k=1}^{n} a_{k, n} \eta_{k}\right|<C_{2}\left(\sum_{k=1}^{n-1}\left|\frac{\eta_{1}+\cdots+\eta_{k}}{k n}\right|+\left|\frac{\eta_{1}+\cdots+\eta_{n}}{n}\right|\right),
$$

где $C_{2}$ - константа, также зависящая только от функции $f(x)$. Согласно условию теоремы, к последовательности взаимно независимых случайных величин $\eta_{n}(n=1,2, \ldots)$ применима первая теорема Колмогорова об усиленном законе больших чисел [4], из которой следует, что с вероятностью 1 справедливо равенство

$$
\lim _{n \rightarrow \infty} \frac{\eta_{1}+\cdots+\eta_{n}}{n}=0 .
$$

Теперь, если предположить, что $\eta_{1}, \ldots, \eta_{n}, \ldots-$ последовательность, для которой справедливо равенство (12), то заключение теоремы следует из неравенства (11) и утверждения, согласно которому если числовая последовательность сходится к нулю, то составленные из ее первых членов средние арифметические также сходятся к нулю. Теорема 4 доказана.

Теорема 5. Пусть выполнень все условия теоремь 4. Тогда с вероятностью 1

$$
\lim _{\substack{n, N \rightarrow \infty \\ N \geqslant n}}\left(\frac{1}{n} \sum_{k=1}^{n} f\left(\frac{k}{N}\right) \xi_{k}-\frac{1}{n} \sum_{k=1}^{n} f\left(\frac{k}{N}\right) M_{k}\right)=0 .
$$

Доказательство теоремы 5 полностью повторяет доказательство теоремы 4.

Теорема 6. Пусть $\xi_{n}(n=1,2, \ldots)$ - бесконечная последовательность взаимно независимых и одинаково распределенных случайных величин, имеюших конечное математическое ожидание $M=\mathbf{E} \xi_{n}$, 
a $f(x)$ - функция на отрезке $0 \leqslant x \leqslant 1$, удовлетворяюшая условию Липшица. Тогда с вероятностью 1 справедливо равенство

$$
\lim _{\substack{n, N \rightarrow \infty \\ N \geqslant n}}\left(\frac{1}{n} \sum_{k=1}^{n} f\left(\frac{k}{N}\right) \xi_{k}-\frac{M}{n} \sum_{k=1}^{n} f\left(\frac{k}{N}\right)\right)=0 .
$$

3 а м е ч а н и е 2. В частном случае, когда $f(x) \equiv 1$, теорема 6 есть вторая теорема Колмогорова об усиленном законе больших чисел [4].

Доказательство теоремы 6 повторяет доказательство теоремы 4 с заменой ссылки на первую теорему Колмогорова ссылкой на вторую теорему Колмогорова.

Основная теорема. Пусть $\xi_{n}(n=1,2, \ldots)$ - бесконечная последовательность взаимно независимьх случайных величин, имеюших конечные математические ожидания $M_{n}=\mathbf{E} \xi_{n}$ u дисперсии $D_{n}=\mathbf{D} \xi_{n}$, удовлетворяющие неравенству $\sum_{n=1}^{\infty} D_{n} / n^{2}<\infty$. Тогда, если для некоторой возрастающей последовательности натуральньх чисел $N$

$$
\varliminf_{N \rightarrow \infty}\left|\frac{1}{N} \sum_{s=1}^{N-1} M_{s} \sin ^{2} \frac{s \pi k}{N}\right|>0
$$

то с вероятностью 1 для этой последовательности чисел $N$ при $N \rightarrow \infty$ справедливо асимптотическое равенство

$$
\omega_{k, N} \sim-\frac{2}{m N} \sqrt{\frac{T}{m}} \sin \frac{\pi k}{2 N} \sum_{s=1}^{N-1} M_{s} \sin ^{2} \frac{s \pi k}{N},
$$

а если $\kappa$ тому же для некоторой возрастающей последовательности натуральных чисел $n$

$$
\varliminf_{\substack{n, N \rightarrow \infty \\ N \geqslant n}}\left|\frac{1}{N} \sum_{s=1}^{n-1} M_{s}\left(\cos \frac{\pi k n}{N}-\cos \frac{2 s \pi k}{N}\right)\right|>0,
$$

то величина $g_{k, N}(n)$ представляется в виде суммы $g_{k, N}(n)=g_{k, N}^{\prime}(n)+$ $g_{k, N}^{\prime \prime}(n)$ так, что с вероятностью 1 для указанных последовательностей чисел $n, N$ nри $n \rightarrow \infty, N \rightarrow \infty, n \leqslant N$ справедливь асимптотические равенства

$$
\begin{aligned}
& g_{k, N}^{\prime}(n) \sim \frac{2 n}{N m} \cos \frac{\pi k n}{N} \operatorname{tg} \frac{\pi k}{2 N} \sum_{s=1}^{N-1} M_{s} \sin ^{2} \frac{s \pi k}{N} \\
& g_{k, N}^{\prime \prime}(n) \sim-\frac{1}{m} \operatorname{tg} \frac{\pi k}{2 N} \sum_{s=1}^{n-1} M_{s}\left(\cos \frac{\pi k n}{N}-\cos \frac{2 s \pi k}{N}\right) .
\end{aligned}
$$

3 а м е ч а н и е 3 . Из Основной теоремы следует, что, несмотря на то, что величины $\omega_{k, N}$ и $g_{k, N}(n)$ являются случайными, при общих предположениях на $\xi_{n}(n \geqslant 1)$ их асимптотическое поведение при $N \rightarrow \infty$, 
$n \rightarrow \infty, n \leqslant N$ и фиксированном значении $k$ уже случайным не является и определяется только математическими ожиданиями случайных величин $\xi_{n}$.

Доказательст в о Основн ой те оремы.

Введем случайные величины

$$
\begin{aligned}
g_{k, N}^{\prime}(n) & =\frac{2 n}{N m} \cos \frac{\pi k n}{N} \operatorname{tg} \frac{\pi k}{2 N} \sum_{s=1}^{N-1} \xi_{s} \sin ^{2} \frac{s \pi k}{N} \\
g_{k, N}^{\prime \prime}(n) & =-\frac{1}{m} \operatorname{tg} \frac{\pi k}{2 N} \sum_{s=1}^{n-1} \xi_{s}\left(\cos \frac{\pi k n}{N}-\cos \frac{2 s \pi k}{N}\right) \\
\alpha_{N} & =\frac{1}{N} \sum_{s=1}^{N-1}\left(\xi_{s}-M_{s}\right) \sin ^{2} \frac{s \pi k}{N} \\
\beta_{n, N}^{\prime} & =\frac{1}{N} \sum_{s=1}^{n-1}\left(\xi_{s}-M_{s}\right) \cos \frac{\pi k n}{N} \\
\beta_{n, N}^{\prime \prime} & =\frac{1}{N} \sum_{s=1}^{n-1}\left(\xi_{s}-M_{s}\right) \cos \frac{2 s \pi k}{N} .
\end{aligned}
$$

Применяя теорему 5 к функциям $f(x)=\sin ^{2}(x \pi k)$ и $f(x)=$ $\cos (2 x \pi k)$, получим равенства

$$
\lim _{N \rightarrow \infty} \alpha_{N}=0, \quad \lim _{\substack{n, N \rightarrow \infty \\ N \geqslant n}} \frac{N}{n} \beta_{n, N}^{\prime \prime}=0,
$$

а согласно первой теореме Колмогорова об усиленном законе больших чисел, с вероятностью 1

$$
\lim _{\substack{n, N \rightarrow \infty \\ N \geqslant n}} \frac{N}{n} \beta_{n, N}^{\prime}=0 .
$$

Далее, в силу (6), (8) и (20) получаем:

$$
\omega_{k, N} \sim-\frac{2}{m N} \sqrt{\frac{T}{m}} \sin \frac{\pi k}{2 N} \sum_{s=1}^{N-1} M_{s} \sin ^{2} \frac{s \pi k}{N}+\frac{2}{m} \sqrt{\frac{T}{m}} \alpha_{N} \sin \frac{\pi k}{2 N},
$$

а в силу $(7),(8),(18),(19),(21)$ и $(22)$ имеем равенство $g_{k, N}(n)=g_{k, N}^{\prime}(n)+$ $g_{k, N}^{\prime \prime}(n)$, в котором

$$
\begin{aligned}
g_{k, N}^{\prime}(n)= & \frac{2 n}{N m} \cos \frac{\pi k n}{N} \operatorname{tg} \frac{\pi k}{2 N} \sum_{s=1}^{N-1} M_{s} \sin ^{2} \frac{s \pi k}{N} \\
& +\frac{2 n}{m} \alpha_{N} \cos \frac{\pi k n}{N} \operatorname{tg} \frac{\pi k}{2 N}, \\
g_{k, N}^{\prime \prime}(n)= & -\frac{1}{m} \operatorname{tg} \frac{\pi k}{2 N} \sum_{s=1}^{n-1} M_{s}\left(\cos \frac{\pi k n}{N}-\cos \frac{2 s \pi k}{N}\right) \\
& -\frac{N}{m}\left(\beta_{n, N}^{\prime}-\beta_{n, N}^{\prime \prime}\right) \operatorname{tg} \frac{\pi k}{2 N} .
\end{aligned}
$$


Поэтому если последовательность чисел $N$ удовлетворяет условию (13), то из (23) и (25) следует справедливость с вероятностью 1 асимптотического равенства (14), а если к тому же последовательность чисел $n$ удовлетворяет условию (15), то из $(23),(24),(26)$ и (27) следует справедливость с вероятностью 1 асимптотических равенств (16) и (17). Основная теорема доказана.

Следствие 1. Пусть $\xi_{n}(n=1,2, \ldots)$ - бесконечная последовательность взаимно независимых случайных величин, имеющих конечнье математические ожидания $M_{n}=\mathbf{E} \xi_{n}$ и дисперсии $D_{n}=\mathbf{D} \xi_{n}$, удовлетворяюшие соотношениям $0 \neq \lim _{n \rightarrow \infty} M_{n}=M<\infty$, $\sum_{n=1}^{\infty} D_{n} / n^{2}<\infty$. Тогда случайньее величиньл $\omega_{k, N}$ nри $N \rightarrow \infty$ свероятностью 1 удовлетворяют асимптотическим равенствам

$$
\omega_{k, N} \sim-\frac{M}{m} \sqrt{\frac{T}{m}} \sin \frac{\pi k}{2 N} .
$$

Д о к а з а т е л ь с т в о. Так как $M_{n} \rightarrow M$ при $n \rightarrow \infty$, то, применяя равенство (14) из утверждения Основной теоремы, при $N \rightarrow \infty$ с вероятностью 1 имеем асимптотическое равенство

$$
\omega_{k, N} \sim-\frac{2 M}{m N} \sqrt{\frac{T}{m}} \sin \frac{\pi k}{2 N} \sum_{s=1}^{N-1} \sin ^{2} \frac{s \pi k}{N} .
$$

Поэтому следствие 1 вытекает из утверждения, согласно которому величина $N^{-1} \sum_{s=1}^{N-1} \sin ^{2}(s \pi k / N)$ есть интегральная сумма для интеграла $\int_{0}^{1} \sin ^{2}(x \pi k) d x$, который равен $\frac{1}{2}$.

Следствие 2. При выполнении условий следствия 1 для любого числа $\beta$, принадлежащего отрезку $0 \leqslant \beta \leqslant 1$, с вероятностью 1 существует предел

$$
\lim _{\substack{n, N \rightarrow \infty \\ n / N \rightarrow \beta}} g_{k, N}(n)=\frac{M \beta}{4 m} \sin (2 \pi k \beta) .
$$

Д о к а з а т е л ь с т в о. Согласно условию следствия 1 и (18)-(24), (26), (27), если $n \rightarrow \infty$ и $\frac{n}{N} \rightarrow \beta \leqslant 1$, то с вероятностью 1

$$
\begin{aligned}
& \lim _{n \rightarrow \infty} g_{k, N}^{\prime}(n)=\frac{\beta \pi k M}{2 m} \cos (\pi k \beta), \\
& \lim _{n \rightarrow \infty} g_{k, N}^{\prime \prime}(n)=-\frac{\beta \pi k M}{2 m} \cos (\pi k \beta)-\int_{0}^{\beta} \cos (2 \pi k y) d y .
\end{aligned}
$$

Поэтому при $n \rightarrow \infty, n / N \rightarrow \beta \leqslant 1$ с вероятностью 1

$$
\lim _{n \rightarrow \infty} g_{k, N}(n)=\lim _{n \rightarrow \infty}\left(g_{k, N}^{\prime}(n)+g_{k, N}^{\prime \prime}(n)\right)=\frac{M \beta}{4 m} \sin (2 \pi k \beta) .
$$


Следствие 3. Пусть $\xi_{n}(n=1,2, \ldots)$ - бесконечная последовательность взаимно независимых и одинаково распределенньх случайньх величин, имеющих конечное математическое ожидание $M=$ $\mathbf{E} \xi_{n} \neq 0$. Тогда с вероятностью 1 при $N \rightarrow \infty$ справедливо асимптотическое равенство (28), а для любого числа $\beta$, принадлежащего отрезку $0 \leqslant \beta \leqslant 1$, справедливо равенство (29).

Д ок а з а т ел ь с т в о. Согласно теореме 6, в этом случае справедливы все утверждения Основной теоремы, ее следствия 1 и 2 , а значит, и следствие 3 .

\section{4. Центральная предельная теорема для частот случайных} колебаний. В этом пункте мы предполагаем, что взаимно независимые случайные величины $\xi_{n}(n \geqslant 1)$, введенные в начале п. 2 , имеют одну и ту же функцию распределения $F(x)$, конечное математическое ожидание $M=\mathbf{E} \xi_{n}$, конечный второй момент $a=\int_{-\infty}^{\infty} x^{2} d F(x)$ и дисперсию $d=\mathbf{D} \xi_{n}$. Результатом же этого пункта является центральная предельная теорема для случайных величин $\omega_{k, N}$, введенных в (8).

Центральная предельная теорема. Прu $N=2,3, \ldots, k=$ $1, \ldots, N-1$ введем числа

$$
M_{k, N}=\frac{2 M}{N m} \sqrt{\frac{T}{m}} \sin \frac{\pi k}{2 N} \sum_{s=1}^{N-1} \sin ^{2} \frac{s \pi k}{N}, \quad D_{k, N}=d \sum_{s=1}^{N-1} \sin ^{4} \frac{s \pi k}{N}
$$

и случайнье величины

$$
\eta_{k, N}=-\frac{m M \sqrt{m / T}}{2 \sqrt{D_{k, N}} \sin (\pi k /(2 N))}\left(\omega_{k, N}-M_{k, N}\right) .
$$

Тогда при Фиксированном $k u N \rightarrow \infty$ распределения вероятностей случайньх величин $\eta_{k, N}$ слабо сходятся $\kappa$ нормальному распределению с плотностью $(2 \pi)^{-1 / 2} e^{-x^{2} / 2}$.

Д ок а з т л ь с т в о. Введем константы

$$
a_{k, N}^{(s)}=\sin ^{2} \frac{s \pi k}{N} \quad(s=1, \ldots, N-1, k=1, \ldots, N-1)
$$

и рассмотрим случайные величины

$$
\xi_{k, N}^{(s)}=\frac{1}{\sqrt{D_{k, N}}} a_{k, N}^{(s)}\left(\xi_{s}-M\right), \quad \widehat{\xi}_{k, N}=\sum_{s=1}^{N-1} \xi_{k, N}^{(s)}
$$

Пусть $\varphi_{k, N}^{(s)}(\lambda)$ - характеристическая функция случайной величины $\xi_{k, N}^{(s)}$, $\varphi_{k, N}(\lambda)$ - характеристическая функция случайной величины $\widehat{\xi}_{k, N}$, а $\varphi(\lambda)$ - характеристическая функция распределения $F(x)$ [4]. Используя взаимную независимость величин $\xi_{n}$ и свойства характеристических 
функций [4], имеем равенство

$$
\varphi_{k, N}(\lambda)=\prod_{s=1}^{N-1}\left(\varphi\left(\frac{\lambda a_{k, N}^{(s)}}{\sqrt{D_{k, N}}}\right) \exp \left(-i \frac{\lambda a_{k, N}^{(s)} M}{\sqrt{D_{k, N}}}\right)\right),
$$

в котором $i-$ мнимая единица. Из определения характеристической функции $\varphi(\lambda)=\int_{-\infty}^{\infty} e^{i \lambda x} d F(x)$ распределения $F(x)$ следует, что $\varphi(\lambda)=$ $1+M i \lambda-\lambda^{2} a / 2+o\left(\lambda^{2}\right)$ при малых $\lambda$, а в силу определения чисел $D_{k, N}$ при больших $N$ справедливо неравенство

$$
D_{k, N}>\widetilde{c} N
$$

где $\widetilde{c}$ - положительная константа, не зависящая от $N$. Поэтому, используя равенство $d=a-M^{2}$ и (31), имеем:

$$
\begin{aligned}
\varphi & \left(\frac{\lambda a_{k, N}^{(s)}}{\sqrt{D_{k, N}}}\right) \exp \left(-\frac{i \lambda a_{k, N}^{(s)} M}{\sqrt{D_{k, N}}}\right) \\
= & \left(1+\frac{i \lambda a_{k, N}^{(s)} M}{\sqrt{D_{k, N}}}-\frac{a \lambda^{2}\left(a_{k, N}^{(s)}\right)^{2}}{2 D_{k, N}}+o\left(\frac{\lambda^{2}}{D_{k, N}}\right)\right) \\
& \times\left(1-\frac{i \lambda a_{k, N}^{(s)} M}{\sqrt{D_{k, N}}}-\frac{\lambda^{2}\left(a_{k, N}^{(s)}\right)^{2} M^{2}}{2 D_{k, N}}+o\left(\frac{\lambda^{2}}{D_{k, N}}\right)\right) \\
= & 1-\frac{\lambda^{2}\left(a_{k, N}^{(s)}\right)^{2} d}{2 D_{k, N}}+o\left(\frac{\lambda^{2}}{N}\right) .
\end{aligned}
$$

Подставляя это равенство в (30), из определений чисел $a_{k, N}^{(s)}$ и $D_{k, N}$ для любого значения $\lambda$ получаем:

$$
\ln \varphi_{k, N}(\lambda)=-\frac{\lambda^{2}}{2}+N o\left(\frac{\lambda^{2}}{N}\right) \rightarrow-\frac{\lambda^{2}}{2} \quad \text { при } N \rightarrow \infty .
$$

Так как $\exp \left(-\lambda^{2} / 2\right)-$ характеристическая функция нормального распределения с плотностью $(2 \pi)^{-1 / 2} e^{-x^{2} / 2}$, то, согласно хорошо известной теореме теории вероятностей, из последнего соотношения следует, что для любого натурального $k$ распределения вероятностей случайных величин $\widehat{\xi}_{k, N}$ при $N \rightarrow \infty$ слабо сходятся к нормальному распределению. Теперь утверждение центральной предельной теоремы сразу следует из определений случайных величин $\eta_{k, N}$ и $\widehat{\xi}_{k, N}$, чисел $M_{k, N}, a_{k, N}^{(s)}$ и из утверждения теоремы 1 , согласно которому справедливы следующие равенства:

$$
-\frac{m N \sqrt{T / m}}{2 \sin (\pi k /(2 N))} \omega_{k, N}=\sum_{s=1}^{N} \xi_{s} \sin ^{2} \frac{s \pi k}{N}, \quad \eta_{k, N}=\widehat{\xi}_{k, N} .
$$

Центральная предельная теорема доказана. 
5. Асимптотическое поведение и центральная предельная теорема для суммы квадратов всех частот случайных колебаний. Как следует из пп. 1 и 2 (теорема С (п. 1) и равенство (5)), общее решение $q_{n}(t)$ уравнения (1) с граничными условиями (2) имеет следующий вид:

$$
q_{n}(t)=\sum_{k=1}^{N-1} c_{k} G_{k, N}(n) \sin \left(\Omega_{k, N} t+\alpha_{k}\right) \quad(n=1, \ldots, N-1),
$$

где $c_{k}, \alpha_{k}(k=1, \ldots, N-1)$ - произвольные константы, зависящие только от начальных данных, $G_{k, N}(n)$ и $\Omega_{k, N}$ - соответственно амплитуда и частота колебаннй, которые являются функциями от масс $m_{n}$ $(n=1, \ldots, N-1)$ при фиксированном значении константы $T$. Введем величину

$$
\Omega_{N}=\sum_{k=1}^{N-1} \Omega_{k, N}^{2}
$$

представляющую собой сумму квадратов всех частот колебаний дискретной струны длины $N-1$. В этом пункте мы находим асимптотику случайной величины $\Omega_{N}$ при $N \rightarrow \infty$ в ситуации, когда массы $m_{n}(n \geqslant 1)$ образуют последовательность положительных взаимно независимых величин (теорема 7 , следствия 4 и 5), и доказываем, что в этом случае последовательность $\Omega_{N}(N=1,2, \ldots)$ удовлетворяет центральной предельной теореме (теорема 8 ).

Теорема 7. Предположим, что при всех $n \geqslant 1$ случайные величины $\eta_{n}=1 / m_{n}$ взаимно независимы, имеют конечные математические ожидания $E_{n}=\mathbf{E} \eta_{n}$ и дисперсии $D_{n}=\mathbf{D} \eta_{n}$, удовлетворяющие неравенствам $\sum_{k=1}^{\infty} D_{k} / k^{2}<\infty u \sum_{k=1}^{n} E_{k}>\bar{c} n$, где константа $\bar{c}>0$ не зависит от $n$. Тогда с вероятностью 1 при $N \rightarrow \infty$ справедливо асимптотическое равенство

$$
\Omega_{N} \sim 2 T \sum_{n=1}^{N-1} E_{n}
$$

Д о к а з а т ел ь с т в о. Применим теорему С из п. 1. Согласно этой теореме, величины $\widetilde{\lambda}_{k}=-\Omega_{k, N}^{2}(k=1, \ldots, N-1)$ являются корнями многочлена $\phi_{N}(\lambda)$ степени $N-1$ и справедливо равенство

$$
\phi_{N}(\lambda)=\widetilde{c} \prod_{k=1}^{N-1}\left(\lambda-\tilde{\lambda}_{k}\right)=\widetilde{c} \prod_{k=1}^{N-1}\left(\lambda+\Omega_{k, N}^{2}\right),
$$

где $\tilde{c}$ - константа, не зависящая от $\lambda$. Разложим $\phi_{N}(\lambda)$ по степеням $\lambda$ так, что

$$
\phi_{N}(\lambda)=\widetilde{c} \sum_{s=0}^{N-1} \phi_{N}^{(s)} \lambda^{s}
$$


$\phi_{N}^{(N-1)}=1, \phi_{N}^{(s)}(s=0, \ldots, N-1)$ - константы, не зависящие от $\lambda$. Согласно формулам Виета и (33), имеем:

$$
\phi_{N}^{(N-2)}=\sum_{k=1}^{N-1} \Omega_{k, N}^{2}
$$

Далее применим равенство (4), из которого следует, что в (33) и (34)

$$
\tilde{c}=\frac{1}{T^{N-1}} \prod_{s=1}^{N-1} m_{s}, \quad \tilde{c} \phi_{N}^{(N-2)}=\frac{2}{T^{N-2}} \sum_{k=1}^{N-1} \prod_{\substack{s=1 \\ s \neq k}}^{N-1} m_{s} .
$$

Поэтому в силу (36) и (35) имеем равенство

$$
\sum_{k=1}^{N-1} \Omega_{k, N}^{2}=2 T \sum_{s=1}^{N-1} \frac{1}{m_{s}}
$$

Согласно условию теоремы 7, к последовательности взаимно независимых случайных величин $\eta_{k}=1 / m_{k}(k \geqslant 1)$ можно применить первую теорему Колмогорова об усиленном законе больших чисел, на основе которой с вероятностью 1

$$
\lim _{n \rightarrow \infty} \frac{1}{n}\left(\sum_{k=1}^{n} \eta_{k}-\sum_{k=1}^{n} E_{k}\right)=0
$$

и, следовательно,

$$
\sum_{k=1}^{n} \frac{1}{m_{k}}=\sum_{k=1}^{n} E_{k}+n \theta_{n}, \quad \lim _{n \rightarrow \infty} \theta_{n}=0 .
$$

Но, согласно условию теоремы 7 , при $n \geqslant 1 \sum_{k=1}^{n} E_{k}>\bar{c} n$, где $\bar{c}>0$. Поэтому в силу (38) при $n \rightarrow \infty$ с вероятностью 1 справедливо равенство $\sum_{k=1}^{n} E_{k}+n \theta_{n} \sim \sum_{k=1}^{n} E_{k}$, и утверждение теоремы 7 непосредственно следует из равенств (32), (37) и (38). Теорема 7 доказана.

Следствие 4. Предположим, что в условиях теоремы 7 существует конечный предел $\widehat{E}=\lim _{n \rightarrow \infty} E_{n}$. Тогда с вероятностью 1

$$
\lim _{N \rightarrow \infty} \frac{\Omega_{N}}{N}=2 T \widehat{E} .
$$

Следствие 5. Предположим, что при всех $n \geqslant 1$ случайные величинь $\eta_{n}=1 / m_{n}$ взаимно независимы, одинаково распределены и имеют конечное математическое ожидание $\widehat{E}=\mathbf{E} \eta_{n}$. Тогда с вероятностью 1 справедливо равенство (39).

Доказательство следствия 5 повторяет доказательство теоремы 7 с заменой в нем ссылки на первую теорему Колмогорова ссылкой на вторую теорему Колмогорова об усиленном законе больших чисел. 
Теорема 8. Предположим, что при $n \geqslant 1$ взаимно независимые случайные величины $\eta_{n}=1 / m_{n}$ имеют одну и ту же функиию распределения $F(x)$, конечное математическое ожидание $\widehat{E}=\mathbf{E} \eta_{n}$, конечный второй момент $\int_{-\infty}^{\infty} x^{2} d F(x)$ и дисперсию $d=\mathbf{D} \eta_{n}$. Тогда $п р и N \rightarrow \infty$ распределения случайньх величин

$$
\frac{1}{\sqrt{4(N-1) T^{2} d}}\left(\Omega_{N}-2(N-1) T \widehat{E}\right)
$$

слабо сходятся $\kappa$ нормальному распределению с плотностью $e^{-x^{2} / 2} / \sqrt{2 \pi}$.

Д о к а з а т е л ь с т в о. Рассмотрим последовательность взаимно независимых и одинаково распределенных случайных величин $\gamma_{n}=2 T \eta_{n}$ $(n \geqslant 1)$, имеющих математическое ожидание $\mathbf{E} \gamma_{n}=2 T \widehat{E}$, дисперсию $\mathrm{D} \gamma_{n}=4 T^{2} d$ и конечный второй момент. Применяя к этой последовательности центральную предельную теорему, получим, что при $N \rightarrow \infty$ распределения случайных величин

$$
\frac{1}{\sqrt{4(N-1) T^{2} d}}\left(\sum_{n=1}^{N-1} \gamma_{N}-2 T(N-1) \widehat{E}\right)
$$

слабо сходятся к нормальному распределению с плотностью $e^{-x^{2} / 2} / \sqrt{2 \pi}$. Поэтому теорема 8 следует из равенств (32) и (37). Теорема 8 доказана.

\section{СПИСОК ЛИТЕРАТУРЫ}

1. Lagrange J. L. Mécanique. V. I. Paris: Gauthier-Villars, 1888.

2. Гантмахер Ф.Р., Крейн М.Г. Осцилляционные матрицы и ядра и малые колебания механических систем. М.: Гостехиздат, 1950, 360 с.

3. Пустьльников Л. Д. О спектре дискретного неоднородного волнового уравнения и колебаниях дискретной струны. - Матем. сб., 1992, т. 183, № 3, с. 38-54.

4. Ширяев А.Н. Вероятность. М.: Наука, 1980, 575 с.

5. Пустыльников Л. Д. Стохастические дискретные волновые уравнения и дискретная струна со случайными массами. Препринт № 7. М.: ИПМ РАН, 1999.

Поступила в редакцию 1.IV.1999 